\title{
Efficient Technique for Improving Data Availability in VANETs
}

\author{
Brij Bihari Dubey ${ }^{1}$, Naveen Chauhan ${ }^{2}$, Narottam Chand ${ }^{3}$ \\ Department of Computer Science and Engineering \\ National Institute of Technology, Hamirpur-177005 (H.P.) \\ Email : $\left\{{ }^{1}\right.$ dubey.brijbihari, ${ }^{2}$ naveenchauhan.nith $\} @$ gmail.com, $\left\{{ }^{3}\right.$ nar $\} @$ nith.ac.in
}

\begin{abstract}
Vehicular networks are useful technique to establish communication using vehicles. Vehicular Delay Tolerant Networks (DTNs) are emerging technology which provides communication among mobile nodes at low cost. In DTN nodes carry data packets till it does not find any neighbor and forwards data to any node moving around other as early as it encounters and agrees to accept that data packet for forwarding. To appease encountered node for data forwarding, requesting node offers incentive to these intermediate nodes if they act as selfish. The network performance gets severely affected due to reluctance shown by selfish nodes. The proposed incentive based mechanism encourages selfish nodes and not only increases mutual cooperation but also reliability in vehicular networks.
\end{abstract}

Keywords—VANETs. Cooperation. Coalition. Incentive. Selfish nodes. Availability.

\section{INTRODUCTION}

Vehicular Ad-hoc Networks (VANETs) are specially design as low cost network which provides communication during mobility. There are several challenges are faced by researchers like fast change in topology among nodes, mobility, reliable channel allocation in limited bandwidth, link establishment and its maintenance due to high mobility in VANETs. In a scenario where nodes having OBU show apathy in actively participating communication and behave as neutral nodes. This reluctance of equipped node is a challenge in the network and has been discussed in recent research works. When in the network, all nodes does not perform in the communication and shows reluctance due to lack of motivation, the network performance degrades severely. To reduce this reluctance we have proposed incentive based scheme to encourage individual node to increase network participation. The proposed scheme not only motivates individual vehicle to participate in the process of data forwarding but also improves fairness in the vehicular network.

In literature, existing works have explored various aspects of $\mathrm{V} 2 \mathrm{~V}$ communication. For instance, in [1] Iqbal et al. proposed framework for traffic congestion and emergency warning message detection. In [2], Yu et al. discussed about safety of vehicular network. In [3], Saad et al. explains the defection function and types of different defection function. It discusses about its applicability to the partition of a network. Saad and Ray [4] discussed about routing authentication security and channel modeling. One challenging aspect in the vehicular network is to circulate data among all nodes as per their requirements by exploiting data exchange capability of nodes. For establishing communication in network Dedicated Short Range Communication (DSRC) guidelines are used in which Federal Communication Consortium (FCC) has allocated $75 \mathrm{MHz}$ of spectrum at the 5.85-5.925 GHz band and implemented the IEEE $802.11 \mathrm{p}$ wireless standard for vehicles to communicate among themselves (V2V communications) and with road-side infrastructure (V2I communication), known as DSRC [5, 6].

There is need to improve performance guarantee. The proposed scheme is based on Reward and Bonus based Incentive (RBI) and motivates to maximize payoff of each individual and of coalition by increasing cooperation among nodes.

The main contributions of this paper is

- To propose an efficient scheme to allocate incentives to forwarding nodes to intermediate nodes to reduce selfishness and increase coalition in the network.

The rest of the paper is organized follows. Section 2 discusses the related works researched in recent years. Section 3 considers few assumptions and explains system model taken in the presented work. Problem statement is defined in the Section 4. Section 5 explains simulation parameters and discusses about performance of incentive based scheme. Finally, Section 6 concludes this paper.

\section{RELATED WORKS}

In recent time many researchers have shown interest in the VANETs. Most of the researches deal with the data dissemination such that more and more data is available with the vehicles and each requesting vehicle receives its requested data with minimum delay. The data availability is improved with the help of lucrative offers suggested to requesting nodes. One of these types of techniques is incentive allocation to each node on data transmission and 
forwarding till it reaches its destination using game theory. In ad hoc network typically following types of schemes are very popular for increasing cooperation among nodes, a) Reputation based scheme, b) Credit based scheme. But all these schemes are not suitable for vehicular network.

\subsection{Reputation based schemes}

Reputation based schemes needs historical data regarding communication between each pair of individual nodes which is complex to manage in case of VANETs due to frequent change in topology because of high mobility of nodes. Nodes are allocated reputation value based on historical cooperation and current trust recommended by their neighbor nodes. Nodes having reputation value less than threshold are penalized by different means. Any node can actively cooperate to avoid punishments. Few reputation based schemes are explained in [7, 8, 9]. These reputation based schemes are not suitable for VANETs, monitoring and identifying those nodes that are not following above criteria, is intricate to estimate due to mobility of nodes.

\subsection{Credit based schemes}

In [10], Zhou et al. discussed about threshold credit based incentive scheme TCBI, which provides secure data forwarding and efficiently reduces resisting layer adding attack. The proposed algorithm securely contains vehicles private information from transportation manager and from the vehicular cloud. In [11], Caballerio et al. proposed time distance and forwarding based scheme to allocate incentives to vehicles forwarding packets to the destination node. Using time factor, author set deadline and allow incentive only if deadline has not expired. Distance parameter sets incentive according to distance. If packet is delivered far away from its source node then its incentive decreased. In [12], Tseng et al. proposed a Reed-Solomon codes (RS-codes) based incentive scheme which enhances security by identifying cooperative vehicle in single operations. The vehicle are allocated private key which is associated with row vector of one RS-code and this private key is used for secure data advertisement purpose. Proposed scheme performs well for disseminating advertisement data using well defined discrete logarithm representation for vehicular networks. In [13], Li et al. analyzed several characteristics of incentive based schemes and by considering incentives for both receiving and forwarding of data. In [14], authors proposed weighted contribution based scheme which finds weight of each node after forwarding data on the basis of number of nodes receiving data. This scheme has drawback of large overhead involved in maintaining record of weights of each node for a data item. In [15], Lee et al. have proposed a scheme based on receipt counting in which the source node forwards data to neighbors and source node claims its reward on the basis of number of nodes that received data. This method suffers with the overspending problem as each forwarding node is allocated fixed rewards without knowing total number of intermediate nodes before forwarding packet to the destination. Incentive based schemes are further divided into two major categories, a) lottery based scheme, b) game theory based scheme.

\section{ASSUMPTIONS}

This section discusses about few assumptions which have been considered in our scenario. We have also described system architecture and design requirements.

\subsection{Assumptions}

Few assumptions taken in this paper are as follows

- Every vehicle gets motivated by reward points and other lucrative offers like-bonus for forwarding private and public data.

- Only one routing protocol is used at a time in the whole network for data transmission.

\section{PROBLEM STATEMENT AND PROPOSED SOLUTION}

In this section, we formulate our problem addressed in this paper.

\subsection{Definitions}

1) Coalition: All the nodes involved in the process of transmitting specific data from source to destination forms a coalition. All nodes in the coalition form a tree known as forwarding tree. 
2) Effort: The nodes which have done effort in forwarding packet in different traffic and network scenarios measured it as effort. It is denoted as $E$. It is one of the parameters to measure total contribution made by each node. 3) Fluke: The nodes which have forwarded packet in different traffic and network scenarios near the destination node. Few of these nodes are supposed to be lucky as they are near to destination and forwaded data to destination and measured it as fluke. It is denoted as $F$. Nodes are awarded incentive on the basis of their fluke contribution also.

4) Contribution: The term contribution $(C)$ is defined as effort $(E)$ and fluke $(F)$ made by any node in forwarding data to destination.

5) Reward: Rewards are the virtual payment made to all the intermediate nodes in the network which are participating in data forwarding.

6) Bonus: Bonus are the virtual payment made to few specific intermediate nodes in the network which are participating in data forwarding and are closer to the destination node.

\subsection{Problem Statement}

In VANETs, the performance of network can be improved when most of the nodes participate in the data forwarding process. The above argument concludes that for better performance of network, there is need of coalition in the network by which each and every node get benefit through lucrative payoff scheme to motivate each node and finally overall network utilizes bandwidth and efficient network is designed.

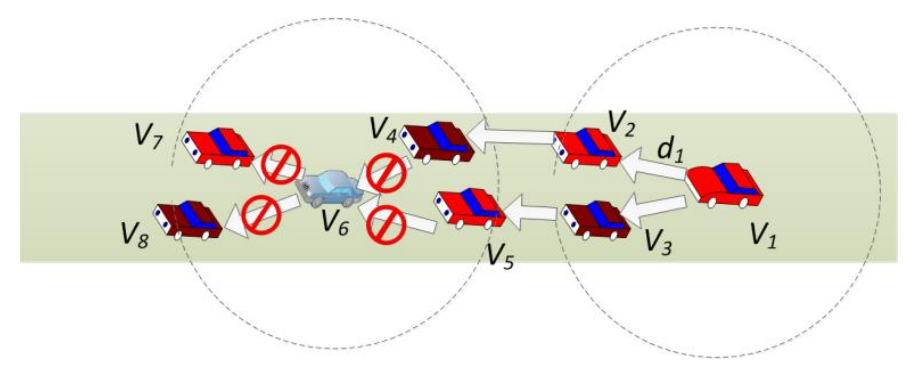

Fig. 1 Scenario of selfish behavior of a node

\subsection{Proposed Solution}

\section{1) Contribution Measurement}

The contribution of node $v_{i}$ for forwarding data $d_{k}$ at any time $t$ is defined by $C_{i}^{c_{j}}\left(t, d_{k}\right)$. Since in any network, total number of intermediate nodes (which participated in communication process) between source and destination can not be accurately predicted until data reaches to destination. In the large network there is high probablility of the fact that packet is forwarded through numerous intermediate nodes during multihop communication. This process reduces the incentive share of each node and may casue nodes to behave desperately and reluctantly in forwarding data. This process degrades performance of network a) by dropping more number of data packets, b) by increasing delay in successful delivery of pakcets. To avoid this situation those nodes which are closer to desination and are awarded fluke $(F)$. The proposed scheme measures total contribution done by those nodes using two paramenters a) Effort, b) Fluke which is defined as

$$
C_{i}^{c_{j}}\left(t, d_{k}\right)=E_{i}^{c_{j}}\left(t, d_{k}\right)+F_{i}^{c_{j}}\left(t, d_{k}\right)
$$

In which, $E_{i}^{c_{j}}\left(t, d_{k}\right)$ is effort involved in successful forwarding of data $d_{k}$ and $F_{i}^{c_{j}}\left(t, d_{k}\right)$ is fluke involved in the same in the coalition $j$ (originated at node other than $v_{i}$ ). Further, Effort is defined as contribution involved in forwarding data from intermediate node to intermediate node or source node. If time elapsed has already crossed the deadline then effort in forwarding data to othe nodes is zero as its deadline already expired. Again, if $d_{i}^{\text {range }}$ is zero then the density in the network . 
Again if,

$$
E_{i}^{c_{j}}\left(t, d_{k}\right)=0 \text { if }\left\{\begin{array}{l}
D_{k}^{e x}-\Delta T \geq 0 \\
o r, d_{i}^{\text {range }}>1 \\
\text { and, } S_{i}^{c_{j}}\left(t, d_{k}\right)=0
\end{array}\right\}
$$

$$
E_{i}^{c_{j}}\left(t, d_{k}\right)=\mu \Delta T \quad \text { if }\left\{d_{i}^{\text {range }}=1\right\}
$$

Otherwise,

$$
\begin{aligned}
& E_{i}^{c_{j}}\left(t, d_{k}\right)= \\
& \alpha\left(\frac{D_{k}^{e x}-\Delta T}{D_{k}^{e x}}\right)+\beta\left(\frac{d_{i}^{\text {region }}}{d_{i}^{\text {range }}}\right)+\gamma\left(\frac{S_{i}^{c_{j}}\left(t, d_{k}\right)}{S_{i}(t)}\right)+\delta^{*} \text { pop }
\end{aligned}
$$

Where, $D_{k}^{e x}$ is the deadline of the data $d_{k}$ which is forwarded from source node. The term $\left(D_{k}^{e x}-\Delta T\right)$ explains the total time for which packet has not been carried by node $v_{i}$.

TABLE I

ALGORITHM OF BONUS

\section{2) Bonus measurement}

Bonus is the additional benefit given the last two vehicles which are closer to the destination. The last two forwarding nodes are; a) intermediate node $v_{4}$ which receives the packet and forwards to; b) intermediate node which is in the range of destination and forwards it to destination (as shown in Fig. 1). The benefit is just acts as lucrative offer for those vehicles which are participating data from source vehicle to the destination vehicle. The bonus provides additional benefit besides rewards. The key point of benefit is that each node forwards data in the hope that it can get additional payoff in terms of bonus but only last and penultimate forwarding vehicles earns bonus points. In the given Fig. 1, node vehicle $v_{1}$ and $v_{2}$ are the source vehicle which generates data $d_{1}$ and $d_{2}$ whose destination is $v_{6}$ and $v_{7}$ respectively. Vehicle $v_{1}$ forwards data $d_{1}$ to its neighbor $v_{2}, v_{3}, v_{4}$. Let Vehicle $v_{2}$ receives data 
$d_{1}$ and forwards first to its neighbors $v_{2}, v_{4}, v_{5}$. Vehicle $v_{5}$ forwards data $d_{1}$ to $v_{4}, v_{7}$ and $v_{6}$. Finally data $v 6$ is received by vehicle $v_{6}$ and last to forwarders are vehicle $v_{3}$ and $v_{5}$ which receives total payoff not only in terms of reward points but also in terms of bonus points.

1) Reasoning for allocating bonus to only last two forwarding nodes (FND and SND): In the coalition there are several nodes including source node, intermediate node and destination node. The last two forwarding nodes are generally selected from the intermediate nodes unless there is only one intermediate node in any coalition.

2) Benefit of bonus: Since bonus is allocated to nodes available at last two adjacent hops to destination then there are following benefits of lucrative offer of bonus

- $\quad$ Nodes earn rewards more than they actually deserve using Equation 1.

- Nodes become willing to store more data to deliver within two hops. This helps in delivering more data before expiring its $D^{e x}$ as $D^{e x}$ is generally larger than it take to transmit at least two hops.

- In the vehicular network probability of getting data in nearby nodes increases.

- Since using Equation 2 as the node expiry comes closure bonus allocated to node also reduces. To boost nodes in that scenario bonus plays important role for forward data.

3) Technique to find FND and SND: For finding last and penultimate forwarding node author have proposed a technique in which each node forwards data with its own ID and also the ID of the node from which it received that data packet.

\section{SIMULATION AND PERFORMANCE ANALYSIS}

\subsection{Simulation model and parameter setup}

We conducted the set of simulations using Network Simulator (NS2) by taking transmission range of the network is to 300 meters. Whenever two nodes enters in the communication range of each other and if these nodes have data ready to forward then both attempts to forward data with probability one only if they are not forwarding any other data in the mean time. The road structure taken is explained in the Fig. 1 and vehicles moves across the road according to the mobility scenario generated with Simulation of Urban MObility (SUMO version 0.23)[16]. Data size varies from $10 \mathrm{~KB}$ to $100 \mathrm{~KB}$. The speed of the node varies between $40-60 \mathrm{~km} / \mathrm{h}$. The default value of $\alpha, \beta$, $\gamma, \delta$ are 0.5 . The buffer size of each node is assumed to be of $2 \mathrm{MB}$ and packets are discarded till the buffer is full. The value of $\kappa$ is set at 2 because to maintain the incentive mechanism lucrative only last two nodes are sufficient to allocated bonus and parameter $\mu$ is set at 0.5 . The value of $\chi$ is set at 1 and time of simulation is set to 1200 seconds. At different network conditions and network requirements the value of above parameters varies and set by the credit center. Network conditions may be different for different times (e.g.- for peak hours), for specific road segments (e.g. road which is one way or have congestion, or suffering with traffic jam due to accident). The simulation parameters are shown in Table II.

TABLE II

SIMULATION PARAMETERS

\begin{tabular}{|l|l|}
\hline Parameters & Values \\
\hline Simulation area & $3000 \times 3000 \mathrm{~m}$ \\
\hline Simulation time & 1200 seconds \\
\hline Number of lanes & 4 \\
\hline Total number of nodes & 50 \\
\hline Transmission range of the vehicles & $300 \mathrm{~m}$ \\
\hline Minimum node speed & $40 \mathrm{~m} / \mathrm{s}$ \\
\hline Maximum node speed & $60 \mathrm{~m} / \mathrm{s}$ \\
\hline Broadcast bandwidth & $115 \mathrm{Kbps}$ \\
\hline Minimum data size & $10 \mathrm{~KB}$ \\
\hline Maximum data size & $100 \mathrm{~KB}$ \\
\hline Zipf distribution parameter $\theta$ & 0.6 \\
\hline
\end{tabular}


We have taken few parameters to measure performance of the proposed incentive based scheme. These parameters are as follows:

1. Average incentive per node in coalition: Incentive received by each node towards forwarding of data.

\subsection{Performance analysis}

For the simulation purpose, it is supposed that when the source node forward data to destination then each node gives its contribution in the range of $(0-40)$.

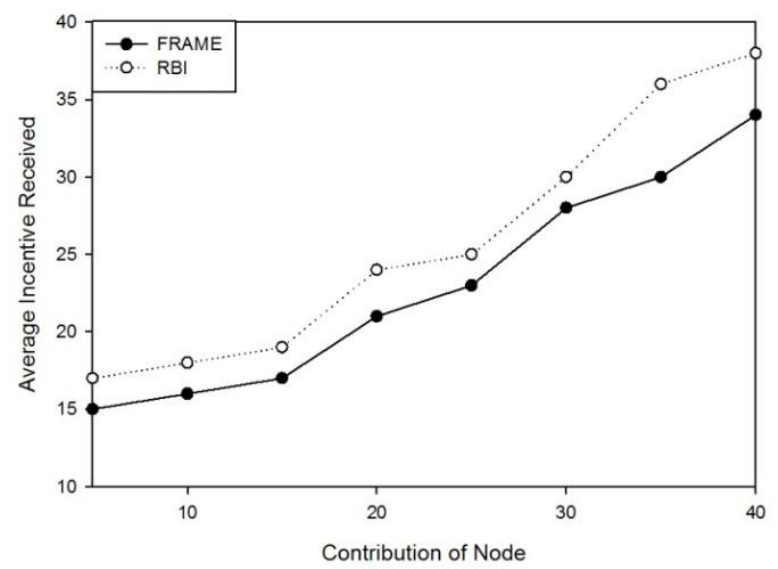

Fig. 2 Plot between average incentives received vs. contribution of node

1) Effect of contribution on Incentive received: When the contribution of nodes increases then the total incentive received by each node increases. Nodes having low incentive due to their effort may have less of more incentive depending upon the bonus points achieved by them due to fluke. Similarly, nodes receiving rewards paid towards their efforts may receive high or low incentive depending upon bonus received due to fluke. In the proposed RBI scheme incentive received by nodes is high as compared to receipt counting as in receipt counting nodes receive only rewards based on total number of nodes and overall motivation was very low (as shown in Fig. 2).

\section{CONCLUSION}

The incentive based schemes are very important to motivate reluctant and selfish vehicles in such a way they get inspired and earn incentives also which are useful to them in requesting other services later. The proposed work finds contribution of each node by taking its effort and fluke into account to measure rewards and bonus points respectively. The incentive fetched by nodes improves as cooperation increases. The simulation shows that random dropping of data decreases incentives earned by nodes. Proposed work provides guarantee to some extent to improve cooperation and data availability among nodes in the network.

In future authors can work on technique which proposes a framework to allocate incentives at the time of successful forwarding of data packet to neighbors or to destination without involving any third party like VCC.

\section{REFERENCES}

[1] Iqbal S., Chowdhury S. R., Hyder C. S. , Vasilakos A. V. \& Wang C., Vehicular communication: protocol design, testbed implementation and performance analysis, In Proceedings of International Conference on Wireless Communications and Mobile Computing, Leipzig, Germany, 2009, 410-415.

[2] Yu F. \& Biswa, S., Self-configuring tdma protocols for enhancing vehicle safety with dsrc based vehicle-to-vehicle communications, In IEEE Journal on Selected Areas in Communications (JSAC), 25(8), 2007, 1526-1537.

[3] Saad W., Han Z., Debbah M., Hjørungnes A. \& Basar T., Coalition game theory for communication networks: A tutorial, In IEEE Signal Processing Magazine., Special issue on Game Theory in Signal Processing and Communications, 26(5), 2009, 77-97.

[4] Ray D., A Game-Theoretic Perspective on Coalition Formation, New York, USA: Oxford University Press, 2007.

[5] Xu Q., Mark T., Ko J. \& Sengupta R., Vehicle-to-vehicle safety messaging in DSRC, In Proceedings of ACM VANET, 2004, 19-28.

[6] Dedicated Short Range Communications (DSRC). [Online].Available:http://grouper.ieee.org/groups/scc32/imwg/index.html 
[7] S. Marti, T. Giuli, K. Lai, and M. Baker, Mitigating routing misbehavior in mobile ad hoc networks, In Proc. MOBICOM00, Boston, MA, Aug. 2000

[8] P. Michiardi and R. Molva, CORE: A collaborative reputation mechanism to enforce node cooperation in mobile ad hoc networks, In Proc. CMS'02 Portoroz, Slovenia, Sep. 2002.

[9] M. T. Refaei, V. Srivastava, L. DaSilva, and M. Eltoweissy, A reputation-based mechanism for isolating selfish nodes in ad hoc networks, In Proc. MobiQuitous'05, San Diego, CA, Jul. 2005.

[10] Zhou J., Dong X., Cao Z., \& Vasilakos A. V., Secure and privacy preserving protocol for cloud-based vehicular DTNs, In IEEE Transactions on Information Forensics and Security, 10(6), 2015, 1299-1314.

[11] Caballero-Gil P., Molina-Gil J., Hernandez-Goya C. \& Caballero-Gil C., Stimulating cooperation in self-organized vehicular networks, In Proceedings of the 15th Asia-Pacific Conference on Communications, 2009, 346-349.

[12] Tseng F.-K., Liu Y.-H., Hwu J.-S. \& Chen R.-J., A secure reed-solomon code incentive scheme for commercial ad dissemination over VANETs, In IEEE Transactions on Vehicular Technology, 60(9), 2009.

[13] Li F. \& Wu J., A winning-probability-based incentive scheme in vehicular networks, In Proceedings of IEEE International Conference on Network Protocols (ICNP), poster abstract, 2008.

[14] Li, F., \& Wu, J. (2009). FRAME: an innovative incentive scheme in vehicular networks. In Proceedings of IEEE International Conference on Communications (ICC).

[15] Lee, S., Pan, G., Park, J., Gerla, M., \& Lu, S. (2007). Secure incentives for commercial ad dissemination in vehicular networks. In Proceedings of ACM MobiHoc.

[16] http://www.dlr.de/ts/en/desktopdefault.aspx/tabid-9883/16931_read-41000/ 